\title{
PCL balancing, an example of the need to couple detailed biomechanical parameters with clinical functional outcome
}

\author{
P. J. C. Heesterbeek • A. B. Wymenga
}

Published online: 28 August 2010

(C) Springer-Verlag 2010

\begin{abstract}
Although the results of a total knee replacement (TKR) are described as being good, many patients are not satisfied after the operation with certain limitations such as the inability to perform sports or other vigorous activities [10, 15]. This motivates engineers and surgeons in their search for superior implant materials and designs, leading to innovations such as surgical navigation and patient-specific cutting blocks, as well as high flex and anatomically formed knee designs. There is even a revival of interest for the bi-cruciate retaining knee implant. Besides these technical topics, the discussion about some more fundamental issues still continues. Still no consensus whether the posterior cruciate ligament (PCL) should be retained or substituted in TKR has been reached. Advocates for both PCL substitution and PCL retention can point to excellent clinical and radiographic results in the literature. Furthermore, findings in the area of biomechanics, histology, and gait analysis do not show convincing evidence for one technique above the other [11]. However, PCL balancing seems to be a factor of major importance. To function properly, the PCL must be accurately tensioned during TKR. This may seem evident, but clinical papers reporting on cruciate-retaining TKRs scarcely report on the functional status of the PCL or the contact point in the operated patients, even though several authors did discuss their finding that PCL insufficiency might be an explanation for some inferior results found for PCL-retaining implants
\end{abstract}

\section{P. J. C. Heesterbeek ( $\square)$}

Research, Development \& Education, Sint Maartenskliniek,

Nijmegen, The Netherlands

e-mail: p.heesterbeek@maartenskliniek.nl

A. B. Wymenga

Department of Orthopaedics, Sint Maartenskliniek,

Nijmegen, The Netherlands
$[4,14]$. Thus far, little scientific data have been published regarding balancing issues related to the PCL, a topic generally considered to be complex.

It seems crucial that the PCL is placed under appropriate tension such that the kinetic benefits of its retention can be enhanced while the adverse effects of either an excessively tight or lax PCL can be avoided. It is essential that a balanced situation is (re)created during TKR since a too tight PCL may result in decreased ROM, potentially affect PE wear, and implant fixation due to posterior loading [12, 13], whereas a too slack PCL may lead to AP instability, which could result in pain, effusion, impaired function, and higher contact stresses [1,13]. But what actually happens during PCL balancing? In two cohort studies, we investigated the relation between flexion gap height and translation of the tibia when the knee was distracted by a tensor. By measuring distances, forces and by performing statistical analyses, we found that balancing the flexion gap is an extremely delicate technique. With a mono-block tensor, the ratio between gap height increase and anterior tibial translation was 1:1.25 [3]. For a bi-compartmental tensor, a higher ratio was found, being 1:1.9. To make it more complex, this ratio was the highest for knees with a steep PCL and with those in which the collateral ligaments had been released during the procedure. In short, the effects of gap distraction were highly variable between patients [7]. Hence, only a $2-\mathrm{mm}$ change in tibia bone cut height, a change of 2-3 degrees in slope of the tibia cut, a change of $2-3 \mathrm{~mm}$ in the bone cut on the posterior condyles of the femur or in PE-insert thickness all can make a difference between a perfectly balanced PCL and one that has been too tightly or too loosely balanced. Without computer navigation, these slight variations are easily made.

When the tibia moves forward during distraction of the flexion gap (whether from the implant, a spacer or a 
tensor), this change in position must have consequences for the tibiofemoral contact point. Fluoroscopic analyses are perfectly suitable to evaluate tibiofemoral contact points after implantation during all functional tasks, but they are not readily available in every clinic and are certainly not feasible in everyday practice for each patient. With a simpler measurement method on plain lateral X-rays in 90 degrees of flexion, a position in which the PCL is most functional, we constructed and validated a measurement technique. This new technique was applied to healthy knees and can be used as a reference for knee implants [5]. The question remains whether a "natural contact point" is ideal for all knee implants or whether the contact point should be at the deepest point of the dished insert used for the specific implant at 90 degrees of flexion. The tibiofemoral contact point is important for the functional outcome since it directly influences the lever arm of the knee during extension. Anterior knee pain could possibly be diminished if during the TKR the knee were perfectly balanced in all directions.

This new knowledge about what happens during flexion gap distraction offers new challenges and possibilities to better balance the PCL during TKR. Probably, the current PCL-retaining TKR with its surgical techniques is not yet as good as it should be. When clinical results of cruciateretaining implants are reported, we encourage clear descriptions of the surgical techniques and also reports on the tibiofemoral contact points in 90 degrees of flexion as well as the AP stability of the knee in this position in order to provide information about whether the PCL was correctly balanced. Such detailed biomechanical measurements, in our opinion, are a prerequisite to permit the critical evaluation of the clinical outcome of a cruciateretaining TKR. Analysing a cohort of patients with an unknown number of incorrectly balanced knees will only obscure the outcome.

To evaluate advancements in new surgical techniques, we do need high quality research focussing on functional outcome. Because today resources for research are not unlimited, it is very important that the money that is invested be well spent. First of all, study designs should be methodologically valid, and the comparisons made in a study should be 'fair'. This may sound evident, but unfortunately this remains a potential pitfall. Furthermore, we need outcome measures that are objective, validated, and sensitive to small changes. Eriksson et al. present an excellent example of more objective measurements in the current issue [6]. Frequently used current knee scoring systems should be evaluated to determine whether they are appropriate to evaluate small design changes; a score that is valuable to evaluate a single patient before and after surgery may be not valid or sensitive enough to evaluate a cohort of patients. Finally, the differences found might be statistically significant, but are they also clinically relevant? When does a result change your practice?

In other studies published in the current issue of KSSTA, the authors also search for superior techniques or for the effect of technical innovations. Lützner et al. did not find a higher functional outcome that could justify the higher costs concerned with navigation [9]. Probably, the KSS is not sensitive enough to detect small functional differences. In another paper, preoperative deformity was identified as significant factor predicting postoperative leg alignment [8]. However, it remains striking that even with good techniques and surgeons, postoperative leg alignment is not optimal in all cases! Furthermore, Cho et al. show that the theoretical advantages of a design do not necessarily translate into a superior outcome [2]. Once again, in addition to just applying new technical material innovations, it is imperative to state what is expected from a new technique or implant.

PCL balancing in TKR is only one example showing the need for more rigorous, detailed biomechanical measurements when reporting the clinical outcome of TKR. Knee laxity and collateral ligament balancing, patellofemoral tracking, and femoral component rotation need to be reported with more biomechanical data that can be linked to the functional outcome. Furthermore, one should check whether the outcomes compare with the proposed specifications of the new technique by making valid comparisons and using validated and accurate measurement techniques. High quality research and a critical analysis of new designs and techniques is the only way to make real advancements in functional outcome after TKR for our patients.

\section{References}

1. Bellemans J, Banks S, Victor J, Vandenneucker H, Moemans A (2002) Fluoroscopic analysis of the kinematics of deep flexion in total knee arthroplasty. Influence of posterior condylar offset. J Bone Joint Surg Br 84:50-53

2. Cho WS, Youm YS, Ahn SC, Sohn DW (2010) What have we learned from LCS mobile-bearing knee system? Knee Surg Sports Traumatol Arthrosc doi:10.1007/s00167-010-1166-5

3. Christen B, Heesterbeek P, Wymenga A, Wehrli U (2007) Posterior cruciate ligament balancing in total knee replacement: the quantitative relationship between tightness of the flexion gap and tibial translation. J Bone Joint Surg Br 89-B:1046-1050

4. Cromie MJ, Siston RA, Giori NJ, Delp SL (2008) Posterior cruciate ligament removal contributes to abnormal knee motion during posterior stabilized total knee arthroplasty. J Orthop Res 26:1494-1499

5. de Jong RJ, Heesterbeek PJ, Wymenga AB (2010) A new measurement technique for the tibiofemoral contact point in normal knees and knees with TKR. Knee Surg Sports Traumatol Arthrosc 18:388-393

6. Eriksson K, Sadr-Azodi O, Singh C, Osti L, Bartlett J (2010) Stress radiography for osteoarthritis of the knee: a new technique. 
Knee Surg Sports Traumatol Arthrosc doi:10.1007/s00167-0101169-2

7. Heesterbeek PJC, Keijsers NLW, Jacobs WCH, Verdonschot N, Wymenga AB (2010) Posterior cruciate ligament recruitment affects antero-posterior translation during flexion gap distraction in total knee replacement. An intraoperative study involving 50 patients. Acta Orthop 81:471-477

8. Hsu WH, Hsu RW, Weng YJ (2010) Effect of preoperative deformity on postoperative leg axis in total knee arthroplasty: a prospective randomized study. Knee Surg Sports Traumatol Arthrosc doi:10.1007/s00167-010-1146-9

9. Lützner J, Günther KP, Kirschner S (2010) Functional outcome after computer-assisted versus conventional total knee arthroplasty: a randomized controlled study. Knee Surg Sports Traumatol Arthrosc doi:10.1007/s00167-010-1153-x

10. Noble PC, Gordon MJ, Weiss JM, Reddix RN, Conditt MA, Mathis KB (2005) Does total knee replacement restore normal knee function? Clin Orthop 431:157-165
11. Pagnano MW, Cushner FD, Scott WN (1998) Role of the posterior cruciate ligament in total knee arthroplasty. J Am Acad Orthop Surg 6:176-187

12. Ritter MA, Faris PM, Keating EM (1988) Posterior cruciate ligament balancing during total knee arthroplasty. J Arthroplasty $3: 323-326$

13. Vail TP, Lang LE (2006) Surgical techniques and instrumentation in total knee arthroplasty. In: Insall JN, Scott WN (eds) Surgery of the knee, 4th edn. Churchill Livingstone Elsevier, Philadelphia, pp 1455-1521

14. Victor J, Banks S, Bellemans J (2005) Kinematics of posterior cruciate ligament-retaining and -substituting total knee arthroplasty: a prospective randomised outcome study. J Bone Joint Surg Br 87:646-655

15. Wylde V, Dieppe P, Hewlett S, Learmonth ID (2007) Total knee replacement: is it really an effective procedure for all? Knee $14: 417-423$ 